\title{
Compositional Invariant Checking for Overlaid and Nested Linked Lists ${ }^{\star}$
}

\author{
Constantin Enea, Vlad Saveluc, and Mihaela Sighireanu \\ Univ Paris Diderot, Sorbonne Paris Cite, LIAFA CNRS UMR 7089, Paris \\ \{cenea, sighirea\}@liafa.univ-paris-diderot.fr, \\ vlad.saveluc@gmail.com
}

\begin{abstract}
We introduce a fragment of separation logic, called NOLL, for automated reasoning about programs manipulating overlaid and nested linked lists, where overlaid means that the lists share the same set of objects. The distinguishing features of NOLL are: (1) it is parametrized by a set of user-defined predicates specifying nested linked list segments, (2) a "per-field" version of the separating conjunction allowing to share object locations but not record field locations, and (3) it can express sharing constraints between list segments. We prove that checking the entailment between two NOLL formulas is co-NP complete using a small model property. We also provide an effective procedure for checking entailment in NOLL, which first constructs a Boolean abstraction of the two formulas in order to infer all the implicit constraints, and then, it checks the existence of a homomorphism between the two formulas, viewed as graphs. We have implemented this procedure and applied it on verification conditions generated from several interesting case studies that manipulate overlaid and nested data structures.
\end{abstract}

\section{Introduction}

Reasoning about behaviors of programs that manipulate dynamic data structures is a challenging problem because of the difficulty of representing (potentially infinite) sets of configurations, and of manipulating these representations for the analysis of the execution of program statements. For instance, pre/post-condition reasoning requires being able, given pre- and post-conditions $\phi$ resp. $\psi$, and a straight-line code $P,(1)$ to compute the (strongest) post-condition of executing $P$ starting from $\phi$, denoted post $(P, \phi)$, and (2) to check that it entails $\psi$. Therefore, an important issue is to investigate logicbased formalisms where pre/post conditions are expressible for the class of programs under interest, and for which it is possible to compute effectively post-conditions, and to efficiently check the entailment. The latter can be done either using theorem provers, where user-provided tactics are needed to guide the proof system, or using decision procedures, when the given annotations are in a decidable fragment. An essential ingredient in order to scale to large programs is being able to perform compositional reasoning and, in this context, Separation Logic [17] (SL) has emerged as a fundamental approach. Its main tool is the frame rule, which states that if the Hoare triple $\{\phi\} P\{\psi\}$ holds and $P$ does not alter free variables in $\sigma$ then $\{\phi * \sigma\} P\{\psi * \sigma\}$ also holds, where $*$ denotes the

\footnotetext{
^ This work has been partially supported by the French ANR project Veridyc and by FSMP.
} 
separating conjunction. Therefore, when reasoning about $P$ one has to manipulate only specifications for the heap region altered by $P$.

In this paper, we define a fragment of SL, called NOLL, suitable for compositional reasoning about programs that manipulate overlaid and nested linked lists, built with an arbitrary set of fields. Such data structures are used in low-level code to link objects with respect to different aspects. For example, the network monitoring software Nagios (www.nagios.com) manipulates hash-tables with closed addressing, implemented as arrays of linked lists, where all the elements in the lists are also linked in the order of their insertion time. Here, we have two overlaid data structures, i.e., which share a set of objects: an array of linked lists and a singly-linked list.

To specify such data structures, NOLL is parametrized by a fixed, but arbitrary, set of recursive predicates defined in a higher-order extension of NOLL and which are expressive enough to specify various types of (nested) linked lists, e.g., singly-linked lists of singly-linked lists, where all the elements point to some fixed object.

To specify that these list segments are overlapped, NOLL includes, besides the classical operator $*$, that we will call object separating conjunction, a field separating conjunction operator $*_{w}$. Both operators separate the heap into disjoint regions, the only difference being the granularity of the separated heap cells. For $*$, a heap cell corresponds to a heap object. For $*_{w}$, a heap cell corresponds to a field from a heap object. Thus, the $*_{w}$ operator allows to specify data structures sharing sets of objects as long as they are built over disjoint sets of fields. In the example above, if ArrOfSI and SI are formulas specifying the array of lists, resp. the list, then ArrOfSI $*_{w} \mathrm{SI}$ expresses the fact that the two structures share some objects.

However, $* w$ alone is not enough to describe precisely overlaid data structures. In the example above, we would also need to express the fact that the objects of the list described by SI are exactly all the list objects in ArrOfSI; let SI_type be their type. To this, we index each atomic formula specifying list segments by a variable, called a set of locations variable and interpreted as the set of all heap objects in the list segment. The values of these new variables can be constrained in a logic that uses classical set operators $\subseteq$ and $\cup$. For example, the specification ArrOfSI $_{\alpha} *_{w} \mathrm{SI}_{\beta} \wedge \alpha($ SI_type $)=\beta$ constrains the set of objects in the linked list to be exactly the set of objects of type SI_type in the array of linked lists. (A NOLL formula $\varphi$ can also put constrains over some set of locations variables, which are not associated to atomic formulas in $\varphi$.)

The semantics of the field separating conjunction $*_{w}$ allows us to establish another frame rule, which is essential for compositional reasoning about overlaid data structures: if the Hoare triple $\{\phi\} P\{\psi\}$ holds then $\left\{\phi *_{w} \sigma\right\} P\left\{\psi *_{w} \sigma\right\}$ also holds, where $P$ is a straight-line code that does not alter fields described by $\sigma$, and the set of locations variables in $\sigma$ are not bound to atomic formulas in $\phi$ or $\psi$. The consequences of this frame rule are that, to reason about a program fragment $P$, one has to provide only specifications for the data structures built with fields altered by $P$.

We prove that checking satisfiability of NOLL formulas is NP-complete and that the problem of checking entailments between NOLL formulas is co-NP complete. The upper bound on the complexity of checking satisfiability/entailment is first proved using a small model argument, and subsequently, following the approach in [8]. The second proof provides also an effective decision procedure for proving the validity of an 
entailment $\varphi \Rightarrow \psi$ by (1) computing a normal form for the two formulas and (2) checking the existence of a homomorphism from the graph representation of the normal form of $\psi$ to the graph representation of the normal form of $\varphi$. The main advantages of this decision procedure are: (i) by defining a Boolean abstraction for NOLL formulas, the construction of the normal form is reduced to (un)satisfiability queries to a SAT solver and (ii) checking the existence of a homomorphism between graph representations of formulas can be done in polynomial time.

To summarize, this work makes the following contributions:

- defines a fragment of SL, called NOLL, that can be used to perform compositional reasoning about overlaid and nested linked structures,

- proves that checking satisfiability, resp. entailment, of NOLL formulas is NPcomplete, resp. co-NP complete,

- defines effective procedures for checking satisfiability and entailment of NOLL formulas based on SAT solvers, which are implemented in a prototype tool and proven to be efficient in practice.

Related Work. SL has been widely used in the literature for the analysis and the verification of programs with dynamic data structures [1] $8,12,13,17,19]$.

The NOLL fragment incorporates several existing features of SL: the separating conjunction $*$ introduced in [12], the separating conjunction $*_{w}$ introduced in [6], and the inductive predicates describing nested linked structures introduced in [1]. The set of location variables are an abstraction of the sequences defined in [17]. However, [1, 6] use these features in order to define abstract domains for program analysis. The (partial) order relation on elements of these abstract domains can be seen as a sound, but not complete, decision procedure for entailment.

The works in [2, 5, 8] introduce results concerning the decidability/complexity of the satisfiability/entailment problem in fragments of SL. Berdine et al. [2] defines a fragment that allows to reason about programs with singly-linked lists and proves that the satisfiability of a formula can be decided in NP and that checking the validity of an entailment between two formulas belongs to the co-NP complexity class. A decision procedure for entailments in the same fragment is introduced in [16], which combines SL inference rules with a superposition calculus to deal with (in)equalities between variables. These complexity results were improved in [8] where it is proved that the satisfiability/entailment problem for the previous fragment can be solved in polynomial time. In fact, the procedure for checking entailments of NOLL formulas based on normal forms and graph homomorphism is inspired by the work in [8]. The differences are that (a) the procedure for computing the normal form of a NOLL formula is based on a new approach that uses Boolean abstractions (the procedure in [8] works only for singly-linked lists and can not be extended to NOLL) and (b) the notion of graph homomorphism is extended in order to handle the two versions of the separating conjunction, the constraints on set of locations variables, and more general recursive predicates.

The (sound) decision procedures for satisfiability/entailment introduced in [18, 15] are also based on Boolean abstractions of formulas. As in our case, the Boolean abstractions are used to transform logical validity into simpler decidable problems. However, they concern different types of logics: algebraic data types specifications for 
reasoning about functional programs in [18] and a recursive extension of first-order logic for reasoning about programs manipulating tree data structures in [15].

Semi-automatic frameworks for reasoning about programs within SL, based on theorem provers, have been defined in [7, 4, 13]. In this paper, we target a completely automatic framework based on decision procedures.

\section{Overview}

In general, $N O L L$ formulas have the form $\Pi \wedge \Sigma \wedge \Lambda$, where $\Pi$ is the pure part, i.e., a conjunction of equalities and inequalities between program variables expressing aliasing constraints, $\Sigma$ is the spatial part specifying the data structures and the separation properties, and $\Lambda$ specifies the sharing constraints between the data structures. The objects building the data structures in the heap are sets of record fields, called simply fields in the following.

$$
\begin{aligned}
\varphi:=x \neq \text { NULL } \wedge \operatorname{Hash}_{\alpha}(x, y, \text { NULL }) *{ }_{w} \text { List }_{\beta}(z, \text { NULL }) \wedge \alpha(\text { SI_type })=\beta \\
\text { Hash }(\text { in }, \text { out }, \text { dest }) \triangleq(\text { in }=\text { out }) \vee(\exists u, v . \text { in } \mapsto\{(g, u) ;(h, v)\} * \text { LowList }(v, \text { dest }) \\
\quad * \text { Hash }(u, \text { out }, \text { dest })) \\
\text { LowList }(\text { in }, \text { out }) \triangleq(\text { in }=\text { out }) \vee(\exists u . \text { in } \mapsto\{(s, u)\} * \text { LowList }(u, \text { out })) \\
\text { List }(\text { in }, \text { out }) \triangleq(\text { in }=\text { out }) \vee(\exists u . \text { in } \mapsto\{(f, u)\} * \text { List }(u, \text { out }))
\end{aligned}
$$

Fig. 1. NOLL specification of a hash table whose elements are shared with a list

Examples of NOLL Formulas: Fig. 1 contains a $N O L L$ formula $\varphi$ describing a list of lists, using the predicate $\operatorname{Hash}_{\alpha}(x, y$, NULL $)$, such that the elements of the nested lists are shared with another list, represented by the predicate $\operatorname{List}_{\beta}(z, \mathrm{NULL})$. This is an abstraction of the hash table sharing all its elements with a singly-linked list, presented in Sec.1] in the sense that we use a linked list to represent the array structure.

The predicate $\operatorname{Hash}_{\alpha}($ in, out, dest $)$ has a recursive definition, written in a higherorder extension of NOLL: either in =out, which means that the nested list segment is empty, or in contains a field $h$ pointing to an inner singly-linked list (in $\mapsto\{\ldots ;(h, v)\} *$ $\operatorname{LowList}(v$, dest $)$ ) and also a field $g$ pointing to a new location $u$ (in $\mapsto\{(g, u) ; \ldots\}$ ), which is the starting point of another nested list segment. Note that the elements of the lists described by LowList (v,dest) are linked by the field $s$. In general, we suppose that variables and fields are typed. Thus, if SI_type is the type of the variables used in the predicate LowList, all the objects in the nested lists are of type SI_type. Moreover, the use of the object separating conjunction $*$ implies that all the nested lists are disjoint.

The overlapping property is expressed using two features of this logic. The first one is the field separating conjunction operator $*_{w}$ which allows to share object locations but not the locations of fields in these objects. The second feature is the ability to speak about the set of all object locations in a list segment. This set of locations is given by the interpretation of the variable that indexes some recursive predicate, e.g., $\alpha$ in $\operatorname{Hash}_{\alpha}(\ldots)$. These variables are constrained in the $\Lambda$ part of a formula. For example, $\alpha($ SI_type $)=\beta$ says that all the locations of type SI_type in the list of lists are also present in the list starting in $z$ ( $\beta$ stands for the set of locations in $\operatorname{List}_{\beta}(z$, NULL $)$ ). 
The operators $*$ and $*_{w}$ can be nested. This is essential to specify a similar data structure (considered in [11]) where the elements stored in a hash table are shared between two disjoint linked lists (using the predicates from Fig. 11):

$$
x \neq \operatorname{NULL} \wedge \operatorname{Hash}_{\alpha}(x, y, \text { NULL }) *_{w}\left(\operatorname{List}_{\beta}(z, \text { NULL }) * \operatorname{List}_{\gamma}(u, \text { NULL })\right) \wedge \alpha(\text { SI_type })=\beta \cup \gamma,
$$

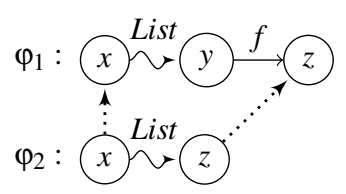

(a)

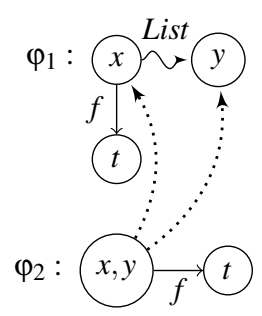

(b)

Fig. 2. where $*$ is used to specify the disjointness of the linked lists starting in $z$ and $u$.

Decision Procedure for Entailment: We define a procedure for checking entailments of NOLL formulas, which is based on the graph homomorphism approach in [8]. The basic idea is to think of formulas as graphs, where nodes represent variables (sets of equal variables) and edges represent list segments, and then, given $\varphi_{1}$ and $\varphi_{2}$ two formulas, if there exists a homomorphism from $\varphi_{2}$ to $\varphi_{1}$ then $\varphi_{1} \Rightarrow \varphi_{2}$ holds. Roughly, the homomorphism is a function mapping each node of $\varphi_{2}$ to a node of $\varphi_{1}$ representing at least the same set of variables. It is required that this function defines a mapping from edges of $\varphi_{2}$ to disjoint paths in $\varphi_{1}$. (Note that the homomorphism is unique.) For example, there exists such a homomorphism from $\varphi_{2}$ to $\varphi_{1}$ in Fig. 2(a), where a snaked edge labeled by List from $x$ to $y$ denotes a predicate $\operatorname{List}(x, y)$, a straight edge labeled by $f$ from $y$ to $z$ denotes a points-to constraint $y \mapsto\{(f, z)\}$, all these constraints are supposed to be separated by $*$, and the dotted edges represent the homomorphism.

In order to be complete, this procedure needs that the formulas of an entailment contain the maximum number of equalities and inequalities; in this case, we say that the formula is in normal form. Also, if it contains an equality $u=v$ then, it contains no spatial constraint defining a list segment from $u$ to $v$ (as usual in separation logic, $u=v \wedge \operatorname{List}(u, v)$ is equivalent to $u=v)$. For example, although the entailment $\varphi_{1} \Rightarrow \varphi_{2}$ in Fig. 2(b) holds, there exists no homomorphism from $\varphi_{2}$ to $\varphi_{1}$. (Because the field $f$ is already defined in $x$, the list segment using this field and starting in $x$ is empty. Thus, $\varphi_{1}$ implies $x=y$, which is needed to show that $\varphi_{1} \Rightarrow \varphi_{2}$.)

Boolean Abstractions of NOLL Formulas: Our first insight in defining such a decision procedure is that the normal form of a NOLL formula $\varphi=\Pi \wedge \Sigma \wedge \Lambda$ can be constructed through a boolean abstraction of $\varphi$, denoted $F(\varphi)$. For the moment, let us consider the case when $\Lambda=$ true. Then, the formula $F(\varphi)$ is defined over a set of boolean variables denoting (in)equalities between variables and atomic formulas from the spatial part $\Sigma$.

We illustrate the definition of $F(\varphi)$ on the formula:

$$
\varphi:=\operatorname{List}(x, y) * \operatorname{List}(x, z) * y \mapsto\{(f, t)\} * \operatorname{List}(y, s) .
$$

The set of boolean variables in $F(\varphi)$ consists of:

- a variable $[u=v]$, for every two variables $u$ and $v$ in $\varphi$,

- a variable $[y, t, f]$ to represent the points-to constraint $y \mapsto\{(f, t)\}$, and

- a variable $[\operatorname{List}(u, v)]$, for every spatial constraint $\operatorname{List}(u, v)$ in $\varphi$. 
In this case, the formula $F(\varphi) \triangleq F_{e q} \wedge F(\Sigma)$, where $F_{e q}$ encodes the reflexivity and the transitivity of the equality relation, i.e.,

$$
\bigwedge_{\text {variables in } \varphi}[u=u] \wedge \quad([u=v] \wedge[v=w]) \Rightarrow[u=w]
$$

and $F(\Sigma)$ models the spatial part of $\varphi$, i.e.,

$$
F(\Sigma) \triangleq[y, t, f] \wedge \bigwedge_{\operatorname{List}(u, v) \text { atom in } \varphi}[\operatorname{List}(u, v)] \oplus[u=v] \wedge \bigwedge_{A, B \text { atoms in } \Sigma} F_{*}(A, B)
$$

The sub-formula $[y, t, f]$ ensures that the points-to constraint is satisfied by any model of $\varphi$; the sub-formula $[\operatorname{List}(u, v)] \oplus[u=v]$ models the fact that in any model of $\varphi$, either $u=v$ or List $(u, v)$ describes a non-empty list segment. The sub-formula $F_{*}(A, B)$ contains the in(equalities) implied by the use of $*$, i.e,

$F_{*}(y \mapsto\{(f, t)\}, \operatorname{List}(u, v)) \triangleq \neg[y=u] \vee[u=v]$, for any $u, v$,

$F_{*}\left(\operatorname{List}\left(u_{1}, v_{1}\right), \operatorname{List}\left(u_{2}, v_{2}\right)\right) \triangleq \neg\left[u_{1}=u_{2}\right] \vee\left[u_{1}=v_{1}\right] \vee\left[u_{2}=v_{2}\right]$, for any $u_{1}, v_{1}, u_{2}, v_{2}$.

In general, the size of $F(\varphi)$ is polynomial in the size of the formula $\varphi$. Also, $\varphi$ is satisfiable iff $F(\varphi)$ is satisfiable.

Computing the Normal Form: The formula $F(\varphi)$ can be used to compute the normal form of $\varphi$ since $\varphi \Rightarrow(u=v)$ iff $F(\varphi) \Rightarrow[u=v]$, for any $u$ and $v$. Thus, for any valid entailment $F(\varphi) \Rightarrow[u=v]$, the equality $u=v$ is added to $\varphi$, and all predicates describing list segments between $u$ and $v$ are removed. For example, the normal form of $\varphi$ in (5) is $y=s \wedge x=z \wedge \operatorname{List}(x, y) * y \mapsto\{(f, t)\}$ (the formula $F(\varphi)$ implies $[y=s]$ and $[x=z]$ ).

Handling Sharing Constraints: For NOLL formulas with sharing constraints, computing the normal form before checking the existence of a graph homomorphism is not enough. Besides (in)equalities, we may have implicit spatial constraints which are not exposed in some formula. Consider the entailment $\varphi_{1} \Rightarrow \varphi_{2}$, where:

$$
\begin{aligned}
& \varphi_{1}:=\operatorname{List}_{\alpha}(x, y) *_{w} \operatorname{LowList}_{\beta}(n, m) \wedge \beta \subseteq \alpha \\
& \left.\varphi_{2}:=\operatorname{List}_{\delta}(x, n) * \operatorname{List}_{\gamma}(n, y)\right) *_{w} \operatorname{LowList}_{\beta^{\prime}}(n, m) \wedge \beta^{\prime} \subseteq \delta \cup \gamma
\end{aligned}
$$

Note that $\beta \subseteq \alpha$ implies that $n$ is a location on the list segment described by $\operatorname{List}_{\alpha}(x, y)$ and thus $\varphi_{1} \Rightarrow \varphi_{2}$ holds. In this case, $F\left(\varphi_{1}\right)$ includes constraints over a set of boolean variables $[u \in \varepsilon]$ representing the fact that $u$ is a location in the set of locations denoted by $\varepsilon$, for any $u$ and $\varepsilon \in\{\alpha, \beta\}$ (we defer the reader to Sec.5 for more details).

In general, if the formula $F(\varphi)$ implies $[u \in \varepsilon]$, for some $u$ and $\varepsilon$, then the graph representation of $\varphi$ includes some additional edges induced by the fact that $u$ is a location on the list segment indexed by $\varepsilon$. In this case, $F\left(\varphi_{1}\right) \Rightarrow[n \in \alpha]$ and the graph representation of $\varphi_{1}$ completed with these additional edges is the graph $\bar{G}\left(\varphi_{1}\right)$ in Fig. 3 Now, it is easy to see that there exists a homomorphism from $G\left(\varphi_{2}\right)$ to $\bar{G}\left(\varphi_{1}\right)$ (the homomorphism must satisfy additional constraints explained in $\mathrm{Sec}$.6.3. 

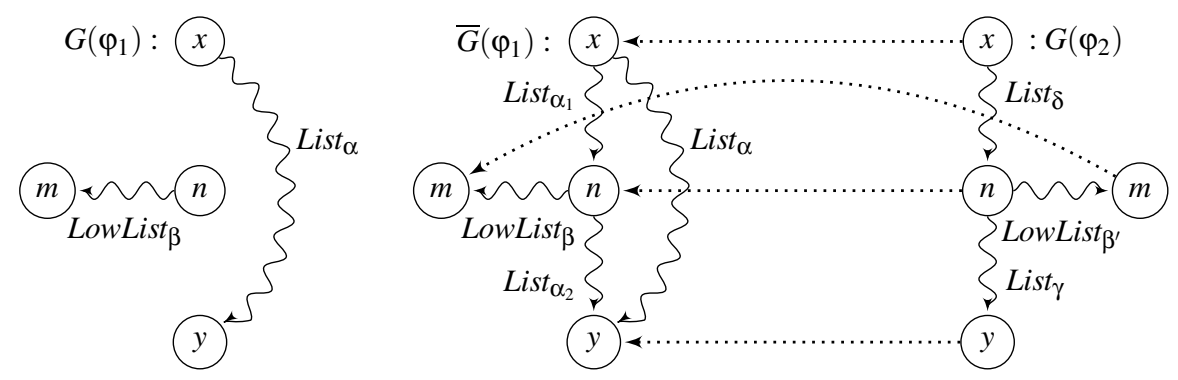

Fig. 3. The graph representations $G\left(\varphi_{1}\right)$ resp. $G\left(\varphi_{2}\right)$ of the (normal forms of the) formulas in eq. (6-7). $\bar{G}\left(\varphi_{1}\right)$ is the graph representation of $\varphi_{1}$ that includes the implicit spatial constraints. Dotted edges represent the homomorphism proving that $\varphi_{1} \Rightarrow \varphi_{2}$.

\section{Logic NOLL}

The logic NOLL is a multi-sorted fragment of Separation Logic [17]. Let $\mathcal{T}$ be a set of sorts (corresponding to record types defined in the program), Flds a set of field names, and $\tau$ a typing function mapping each field name into a function type over $\mathcal{T}$. A field $f \in$ Flds is called recursive iff $\tau(f)=R \rightarrow R$ with $R \in \mathcal{T}$ and non-recursive, otherwise. The set of recursive fields is denoted by Flds $s_{\text {rec }}$.

Syntax: Let LVars and SetVars be two sets of variables, called location variables and set of locations variables, respectively. We assume that the typing function $\tau$ associates a sort, resp. a set of sorts, to every variable in LVars, resp. SetVars. For simplicity, we assume that LVars contains the constant NULL. The syntax of NOLL is given in Fig. 4.

$$
\begin{aligned}
& \begin{aligned}
x, y, y_{i} \in \text { LVars } & \text { location variables } & \vec{z} \in \text { LVars }^{+} & \text {tuples of location variables } \\
f, f_{i} \in \text { Flds } & \text { field names } & \alpha \in \text { SetVars } & \text { set of locations variables } \\
R \in \mathcal{T} & \text { sort } & P \in \mathcal{P} & \text { list segment predicates }
\end{aligned} \\
& \varphi::=\Pi \wedge \Sigma \wedge \Lambda \quad \text { NOLL formula } \\
& \Pi::=\text { true }|x \neq y| x=y \mid \Pi \wedge \Pi \quad \text { pure constraints } \\
& \Sigma::=e m p\left|x \mapsto\left\{\left(f_{1}, y_{1}\right) ; \ldots ;\left(f_{k}, y_{k}\right)\right\}\right| P_{\alpha}(x, y, \vec{z})|\Sigma * \Sigma| \Sigma *_{w} \Sigma \quad \text { spatial constraints } \\
& \Lambda::=\text { true }\left|t \subseteq t^{\prime}\right| x \in t|x \notin t| \Lambda \wedge \Lambda \quad \text { sharing constraints } \\
& t::=\{x\}|\alpha| \alpha(R) \mid t \cup t^{\prime} \quad \text { set of locations terms }
\end{aligned}
$$

Fig. 4. Syntax of NOLL formulas

An atomic points-to constraint $x \mapsto\left\{\left(f_{1}, y_{1}\right) ; \ldots ;\left(f_{k}, y_{k}\right)\right\}$ is used to specify the values of fields $f_{1}, \ldots, f_{k}$ in the location denoted by $x$ : the value stored by the field $f_{i}$ is $y_{i}$, for all $1 \leq i \leq k$. The fields shall be pairwise disjoint and the formula shall be well typed, i.e., for any $f_{i}, \tau\left(f_{i}\right)=\tau(x) \rightarrow \tau\left(y_{i}\right)$.

In every list segment constraint $P_{\alpha}(x, y, \vec{z}), P$ is a predicate from a fixed, but arbitrary, set $\mathcal{P}$. The predicates in $\mathcal{P}$ have recursive definitions with the following syntax: 


$$
\begin{aligned}
P(\text { in }, \text { out }, \overrightarrow{n h b}) \triangleq & (\text { in }=\text { out }) \vee \\
& \left(\exists u, \vec{v} \cdot \Sigma_{0}(\text { in }, u \cup \vec{v} \cup \overrightarrow{n h b}) * \Sigma_{1}(\vec{v}, \overrightarrow{n h b}) * P(u, \text { out }, \overrightarrow{n h b})\right) \\
\Sigma_{0}(\text { in }, V)::= & \text { in } \mapsto \theta, \text { where } \theta \subseteq\{(f, w) \mid f \in F l d s, w \in V\} \\
\Sigma_{1}(\vec{v}, \overrightarrow{n h b})::= & \text { emp }|Q(v, b, \vec{b})| \Sigma_{1}(\vec{v}, \overrightarrow{n h b}) * \Sigma_{1}(\vec{v}, \overrightarrow{n h b}) \text { with } b, \vec{b} \subseteq \overrightarrow{n h b}, \text { and } Q \in \mathcal{P}
\end{aligned}
$$

where in, out, $u \in$ LVars and $\overrightarrow{n h b}, \vec{v}, \vec{b} \in$ LVars $^{+}$. The definition of every $P \in \mathcal{P}$ is well typed and satisfies the additional typing constraints $\tau($ in $)=\tau($ out $)=\tau(u)$, and $\tau($ in $) \neq \tau(v)$, for every $v \in \vec{v}$. Moreover, the definitions in $\mathcal{P}$ are not mutually recursive.

A predicate $P($ in, out, $\overrightarrow{n h b})$ defines possibly empty list segments starting from in and ending in out. The fields of each element in this list segment are defined by $\Sigma_{0}$ while the nested lists to which it points to are defined by $\Sigma_{1}$. The parameters $\overrightarrow{n h b}$ are used to define the "boundaries" of the nested list segment described by $P$, in the sense that every location described by $P$ belongs to a path between in and some location in out $\cup \overrightarrow{n h b}$ (this path may be defined by more than one field). Every element of the list segment described by $P$ points to several nested lists, each one of them being described by a predicate $Q$ in $P$. The use of $*$ in the definition of $P$ implies that the inner list segments are disjoint. The typing constraints ensure bounded nesting.

For simplicity of the presentation, we have restricted ourselves to such inductive definitions, which are not expressive enough to describe doubly-linked lists or nested lists containing cyclic lists on their inner levels. However, our techniques can be extended to cover such cases. For example, to describe doubly-linked lists, one must allow further points-to constraints and use a special type of existential variables representing the next to last location in a doubly-linked list segment like, e.g., in [1].

For any predicate $P, \Sigma_{0}(P)$, resp. $\Sigma_{1}(P)$, denotes the sub-formula $\Sigma_{0}$, resp. $\Sigma_{1}$ of $P$. Moreover, $F l d s_{0}(P)$ denotes the set of fields of $i n$ that point to $u$ according to the formula $\Sigma_{0}(P)$, i.e., $f \in$ Fld $_{0}(P)$ iff $\Sigma_{0}(P)=i n \mapsto \theta$ and $(f, u) \in \theta$.

In every spatial constraint $P_{\alpha}(x, y, \vec{z}), \alpha$ is a set of locations variable, which is said to be bounded to or to index the spatial constraint. The constraint $\Lambda$ may contain set of locations variables which are not bounded to some spatial constraint. For simplicity, we assume that a variable in SetVars appears in $\Sigma$ at most once. Also, we consider that all atomic constraints in $\Lambda$ are well typed, i.e., for any $t \subseteq t^{\prime}$ in $\Lambda, \tau(t) \subseteq \tau\left(t^{\prime}\right)$ and for any $(x \in t)$ in $\Lambda, \tau(x) \in \tau(t)$, where $\tau$ is extended to set of locations terms as usual.

In the following, we denote by $\operatorname{LVars}(\varphi)$ (and $\operatorname{Set} \operatorname{Vars}(\varphi)$ ) the set of location variables (resp. set of locations variables) used in $\varphi$. Also, atoms $(\varphi)$ denotes the set of atomic formulas in $\varphi$. Two atoms in $\Sigma$ are object separated, resp. field separated, if their least common ancestor in the syntactic tree of $\varphi$ is $*$, resp. $*_{w}$.

Semantics: Let Loc be a multi-sorted set of locations typed by the typing function $\tau$, and let $L o c_{R}$ denote the set of locations in $L o c$ of sort $R$.

A program heap is modeled by a pair $C=(S, H)$, where $S:$ LVars $\rightarrow$ Loc maps location variables to locations in $L o c$ and $H: L o c \times F l d s \rightarrow L o c$ defines values of fields for a subset of locations. Intuitively, each allocated object is denoted by a location in $L o c$ and then, $H$ defines the fields for the allocated objects and $S$ gives for each variable, the object it points to. The set of locations $l$ for which there exists $f$ s.t. $H(l, f)$ is defined is called the set of locations in $C$, and denoted by $\operatorname{Loc}(C)$. The component $S$ (resp. $H$ ) of a heap $C$ is denoted by $S^{C}$ (resp. $H^{C}$ ). 


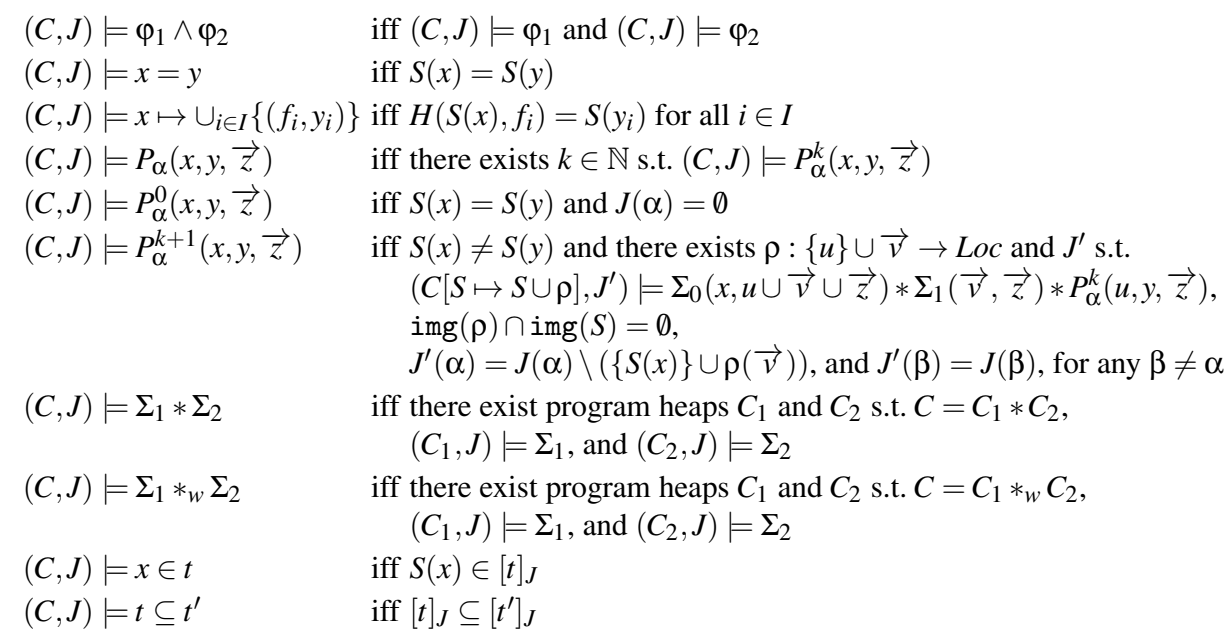

Separation operators over program heaps:

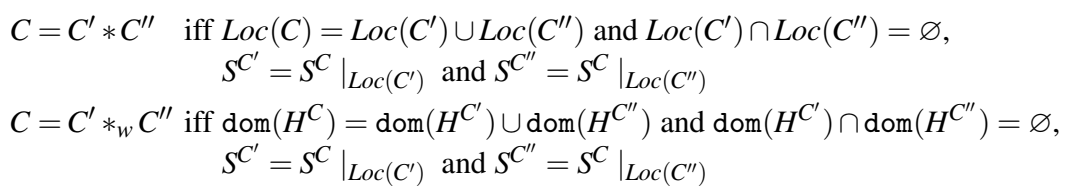

Interpretation of a term $t,[t]_{J}$ :

$[\{x\}]_{J}=\{S(x)\}, \quad[\alpha]_{J}=J(\alpha), \quad[\alpha(R)]_{J}=J(\alpha) \cap \operatorname{Loc}_{R}, \quad\left[t \cup t^{\prime}\right]_{J}=[t]_{J} \cup\left[t^{\prime}\right]_{J}$.

Fig. 5. Semantics of $N O L L$ formulas. $\operatorname{dom}(F)$ denotes the domain of the function $F$ and $S \cup \rho$ denotes a new mapping $K: \operatorname{dom}(S) \cup \operatorname{dom}(\rho) \rightarrow L o c$ s.t. $K(x)=\rho(x), \forall x \in \operatorname{dom}(\rho)$ and $K(y)=$ $S(y), \forall y \in \operatorname{dom}(S))$.

NOLL interpretations are pairs $(C, J)$, where $C=(S, H)$ is a program heap and $J$ : SetVars $\rightarrow 2^{L o c}$ interprets variables in SetVars to finite subsets of Loc. We assume that $S, H$, and $J$ are well-typed w.r.t. $\tau$. A $N O L L$ interpretation $(C, J)$ is a model of a formula $\varphi$ iff $(C, J) \models \varphi$, where $\models$ is defined in Fig. 5 for its non trivial cases. For simplicity, we consider the intuitionistic semantics of SL [17]: if a formula is true on a model then it remains true for any extension of that model with more locations. Our techniques can be adapted to work also for the non-intuitionistic semantics [10].

Note the difference between the two kinds of separation of heaps: $C=C^{\prime} * C^{\prime \prime}$ holds iff the set of locations in $C^{\prime}$ and $C^{\prime \prime}$ are disjoint while $C=C^{\prime} *_{w} C^{\prime \prime}$ holds iff the domains of the $H$ component in $C^{\prime}$ and $C^{\prime \prime}$ are disjoint.

W.l.o.g., we suppose that the sharing constraints in $\Lambda$ are in a simplified form obtained as follows. First, inclusion constraints are put in the form $\alpha \subseteq t$, where $t$ contains at most two set of locations variables. Second, for any atomic formula $\alpha \subseteq t$ in $\Lambda$ such that $\alpha$ is bound to some spatial constraint $P_{\alpha}(x, y, \vec{z})$, we remove from $t$ (1) all the variables $\alpha^{\prime}$ such that $\alpha$ and $\alpha^{\prime}$ are bound to object separated spatial constraints and (2) all the terms of the form $\{x\}$ such that $\varphi$ contains a points-to constraint $x \mapsto \theta$, which is object separated from the spatial constraint indexed by $\alpha$. If $t$ becomes empty then, the equality $x=y$ is added to $\varphi$. 
We denote by $[\varphi]$ the set of pairs $(C, J)$ which are models of $\varphi$. The entailment between two NOLL formulas is denoted by $\Rightarrow$ and it is defined by $\varphi \Rightarrow \psi$ iff $[\varphi] \subseteq[\psi]$.

Fragment $M O L L$ : To illustrate some constructions in this paper, we consider the fragment MOLL which does not allow to specify nested lists, but only overlaid multilinked lists. Formally, the fragment MOLL contains all the NOLL formulas defined over a set of predicates $\mathcal{P}$ such that, for any $P \in \mathcal{P}, \Sigma_{1}(P)=e m p$, i.e., $P$ is defined by $P($ in, out, $\overrightarrow{n h b}) \triangleq($ in $=$ out $) \vee\left(\exists u . \Sigma_{0}(\right.$ in,$u \cup \overrightarrow{n h \vec{b}}) * P(u$, out, $\left.\overrightarrow{n h \vec{b}})\right)$.

\section{A Model-Theoretic Procedure for Checking Entailment}

We prove that satisfiability, resp. entailment checking, of NOLL formulas is NPcomplete, resp. co-NP complete. The upper bound for the complexity of satisfiability is proved using a small model property: if $\varphi \in N O L L$ has a model, then it has also a model of size polynomial in the size of $\varphi$ and $\mathcal{P}$ (the size of $\mathcal{P}$ is defined as the size of all recursive definitions for predicates in $\mathcal{P}$ ). The co-NP upper bound for entailment checking is obtained by proving a small model property for formulas of the form $\varphi \nRightarrow \psi$ (a model for this formula corresponds to a counter-example for $\varphi \Rightarrow \psi$ ).

\subsection{Satisfiability Problem}

The NP lower bound of the satisfiability problem for NOLL formulas is given by the next theorem. The proof is based on a reduction of 3SAT, the satisfiability problem for CNF formulas with three literals in each clause, to the satisfiability problem for MOLL formulas. The proof of this result is detailed in [10].

Theorem 1. The satisfiability problem for NOLL (MOLL) is NP-hard.

To prove the small model property for the NP upper bound, we use an abstraction of the models of NOLL formulas by colored heap graphs. Intuitively, a model $(C, J)$ of a NOLL formula is represented by a colored graph where each location $\ell$ from $C$ is represented by a set of graph nodes $V_{\ell} . V_{\ell}$ is a singleton when $\ell$ is the interpretation of a location variable or it is not shared between list segments described in $\varphi$. Otherwise, each node in $V_{\ell}$ represents a subset of fields at location $\ell$ such that two nodes in $V_{\ell}$ represent disjoint sets of fields. All nodes in $V_{\ell}$ are colored by $\ell$ and are called sibling nodes. The abstraction is built such that the sub-graphs corresponding to list segments defined using different atoms of $\varphi$ share only nodes which are interpretations of location variables. Thus, we can collapse in these sub-graphs most of nodes and still obtain a model of $\varphi$. The collapsed nodes shall not be colored by the interpretation of a location variable, i.e., they are anonymous nodes. We show that for any model $(C, J)$, one can identify a set of anonymous nodes, whose size is polynomial in the size of $\varphi$ and $\mathcal{P}$, called crucial nodes, such that by collapsing all the non-crucial anonymous nodes one can still obtain a model of $\varphi$. Formally, 
Definition 1 (Colored heap graph). A colored heap graph over LVars, Flds, and SetVars is a tuple $G=(V, E, \mathcal{P}, \mathcal{L}, \mathcal{S})$, where (1) $V$ is a finite set of nodes, (2) $E$ : $V \times$ Flds $\rightarrow V$ is a set of edges, (3) $\mathcal{P}: \operatorname{LVars}(\varphi) \rightarrow V$ is a labeling of nodes with location variables, (4) $\mathcal{L}: V \rightarrow$ Loc is a coloring of nodes with locations, and (5) $\mathcal{S}:$ SetVars $\rightarrow 2^{V}$ is an interpretation of variables in SetVars to sets of nodes.

Fig. 6pictures a model of $\varphi$ in eq. (1) and its colored heap graph abstraction. We denote the components of a colored heap graph $G$ using superscripts, e.g., the set $V$ in $G$ is denoted by $V^{G}$. The semantics of NOLL formulas on colored heap graphs is defined similarly to the one on NOLL interpretations, except for $*$ and the constraints in $\Lambda$. A colored heap graph $G$ satisfies a formula $\varphi_{1} * \varphi_{2}$ iff $G$ can be split into two disjoint graphs $G_{1}$ and $G_{2}$ such that $G_{1} \models \varphi_{1}, G_{2} \models \varphi_{2}$, and for any two nodes $v_{1} \in V^{G_{1}}$ and $v_{2} \in V^{G_{2}}, \mathcal{L}^{G_{1}}\left(v_{1}\right) \neq \mathcal{L}^{G_{2}}\left(v_{2}\right)$. Also, for any constraint $P_{\alpha}(x, y, \vec{z}), \mathcal{S}(\alpha)$ is interpreted as the union of $\mathcal{L}(v)$, for all nodes $v$ in the unique subgraph defined by $P_{\alpha}$.

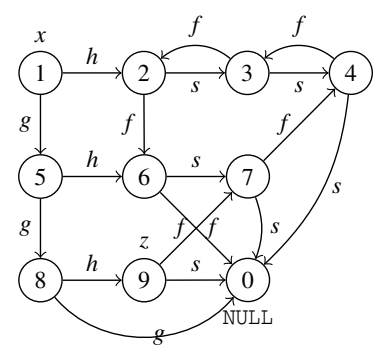

(a)

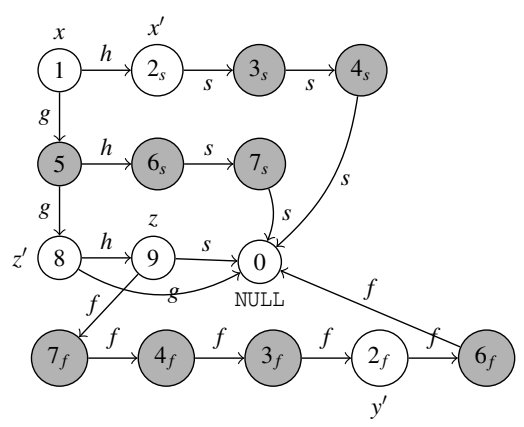

(b)

Fig. 6. A program heap satisfying $\varphi$ in (1) and its colored heap graph. For any $0 \leq n \leq 9$, the nodes $n_{s}$ and $n_{f}$ in (b) are colored by the location $n$ from (a). Primed variables $x^{\prime}, y^{\prime}, z^{\prime}$ label crucial nodes. A small model is obtained by collapsing filled nodes in (b).

Lemma 1. If a NOLL formula $\varphi$ has a model $(C, J)$ then it also has a model $\left(C_{S}, J_{S}\right)$ of size polynomial in the size of $\varphi$ and $\mathcal{P}$.

Proof. (Idea) The proof builds a small model following the steps given in Fig. 7a, Roughly, we show that anonymous locations from $(C, J)$ can be collapsed until the list segments are of bounded length. The bounds are determined by the sharing constraints in $\varphi$ and the levels of nesting in the definition of the recursive predicates. To collapse anonymous locations on list segments, we use the colored heap graph abstraction. However, some distinguished set of crucial anonymous nodes shall not be collapsed because this will invalidate spatial or sharing constraints in $\varphi$ (an example is shown below). Also, to preserve the truth value of sharing constraints, if a node is found crucial on some list segment, then all its sibling nodes are also marked as crucial (this corresponds to the fact that the small model contains all the fields for that location). 
The procedure purify removes from $(C, J)$ all the locations not involved in spatial constraints from $\varphi$. This is possible because the minimal part of $C$ satisfying some spatial constraint is unique. splitLocations builds the colored heap graph abstraction of $\left(C^{\prime}, J^{\prime}\right)$ by splitting the nodes not labeled by location variables but shared between several list segments described by predicates in $\varphi$. An example is given in Fig. 6

$$
\begin{array}{ll}
\text { 1: } & \left(C^{\prime}, J^{\prime}\right):=\operatorname{purify}(\varphi)(C, J) \\
\text { 2: } & G:=\operatorname{splitLocations}\left(C^{\prime}, J^{\prime}\right) \\
\text { 3: } & V^{\prime}:=\operatorname{crucialNodes}(\varphi, G) \\
\text { 4: } & G^{\prime}:=\text { labelCrucial }\left(G, V^{\prime}\right) \\
\text { 5: } & G^{\prime \prime}:=\operatorname{collapseAnonymous}\left(G^{\prime}\right) \\
\text { 6: } & \left(C_{S}, J_{S}\right):=\operatorname{mergeNodes}\left(G^{\prime \prime}\right)
\end{array}
$$

(a) Steps for computing a small model
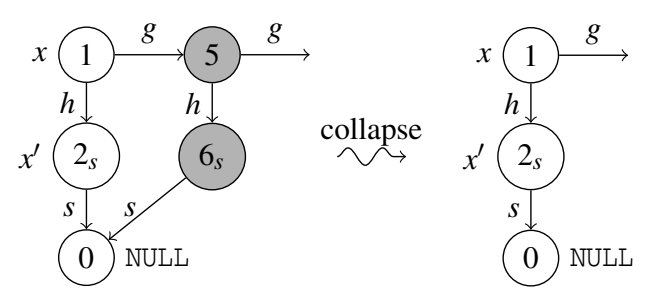

(b) Example of collapsing

Fig. 7. Computing a small model for NOLL formulas

crucialNodes computes the set of crucial nodes $V^{\prime}$ as the closure under the sibling relation of the set of (anonymous) nodes in $G$ which are either (1) the successor of a labeled node by a non recursive field (e.g., node $2_{s}$ in Fig. 6), or (2) the source or the target of a non recursive field on a fixed path between two nodes labeled by location variables (e.g., node 8 in Fig. 6. Because the nesting of recursive predicates is bounded, the size of the set $V^{\prime}$ is bounded by a polynomial in the size of $\varphi$ and $\mathcal{P}$ (the number of variables, the nesting depth, and the size of Flds). The crucial nodes are labeled with a set of additional location variables LVars $^{\prime}$ in labelCrucial.

Afterwards, the anonymous nodes (not labeled by variables in $\operatorname{LVars}(\varphi) \cup \operatorname{LVars}^{\prime}$ ) are collapsed by collapseAnonymous in a bottom up manner, i.e., starting from the inner list segments to the upper ones. Roughly, the collapsing removes a node (and the sub-graph representing the nested, anonymous structure) if it is between two recursive fields (see Fig.7b). Intuitively, this process preserves a model of $\varphi$ because no edges are added and the nodes marked as important for the satisfaction of the spatial and sharing constraints are kept. Due to the special syntax of predicates in $\mathcal{P}$, we can compute for each list segment the minimal number of anonymous nodes that must be preserved in order to satisfy some given spatial constraint. This number depends only on the size of $\mathcal{P}$ and it is obtained when all the spatial constraints in the predicate definition are interpreted as list segments of length one. Thus, we obtain a colored heap graph $G^{\prime \prime}$ where all labeled nodes are preserved and with them some sub-graphs with a bounded number of anonymous nodes. Finally, from $G^{\prime \prime}$, a (small) model $\left(C_{s}, J_{s}\right)$ of $\varphi$ is built, by applying mergeNodes, which roughly merges sibling nodes in locations.

Since the complexity of the model-checking problem for NOLL formulas is polynomial, the following result holds.

Theorem 2. The satisfiability problem for NOLL is NP-complete. 


\subsection{Entailment Problem}

The colored heap graph abstraction is also used to prove a small counter-example property for entailments $\varphi \Rightarrow \psi$ when $\varphi$ and $\psi$ are in NOLL. The proof is similar to the proof of Lemma 1, with two main differences. Let $(C, J)$ be a counter-example for $\varphi \Rightarrow \psi$. First, in purify, the locations not used in $\varphi$ are removed from $(C, J)$ except for locations that are witnesses for some unsatisfied sharing constraint in $\psi$. It is enough to keep one location per sharing constraint in $\psi$ and thus, their number is bounded by the size of $\psi$. We label these locations with variables from some set VVars $^{\prime \prime}$. Second, crucialNodes marks some additional nodes as crucial, in order to keep track if two list segments are sharing at least one location and in order to distinguish between list segments of size 1 and list segments of size at least 2 . However, this process adds at most one more node per constraint, and thus the bound on the number of nodes is increased by a linear term in the size of $\varphi$ and $\psi$. This property and the NP-completeness of satisfiability imply:

Theorem 3. Checking the validity of an entailment between two NOLL formulas is coNP complete.

\section{Computing the Normal Form}

This section makes a first step towards the effective procedure for checking entailments of NOLL formulas by presenting the procedure for computing the normal form of a $N O L L$ formula. We say that a NOLL formula is in normal form if it contains the maximum set of equalities and disequalities between location variables and the minimum set of list segment constraints. Formally,

Definition 2 (Normal form). A NOLL formula $\varphi=\Pi \wedge \Sigma \wedge \Lambda$ is in normal form iff:

- for any $x, y \in \operatorname{LVars}(\varphi)$, if $\varphi \Rightarrow x=y$, resp. $\varphi \Rightarrow x \neq y$, then $\Pi$ contains the atom $x=y$, resp. $x \neq y$, and

- for any atomic formula $P_{\alpha}(x, y, \vec{z})$ in $\Sigma$, there exists a model $(C, J)$ of $\varphi$ such that $S^{C}(x) \neq S^{C}(y)$.

The normal form of $\varphi$ is a formula $\varphi^{\prime}$ in normal form and equivalent to $\varphi$.

We now describe the main ideas behind the procedure that computes the normal form and to this, we must define the class of reduced, explicit NOLL formulas.

A NOLL formula is called explicit if it contains $x=y$ or $x \neq y$, for any constraint $P_{\alpha}(x, y, \vec{z})$ in $\varphi$, and $x \in \alpha$ or $x \notin \alpha$, for any $x$ and $\alpha$ in $\varphi$. Then, an explicit formula $\psi$ is called reduced if it does not contain both the atoms $x=y$ and $P_{\alpha}(x, y, \vec{z})$.

Any NOLL formula $\varphi$ is equivalent to a disjunction of reduced, explicit formulas $\psi_{1} \vee \ldots \vee \psi_{n}$. The formulas $\psi_{i}$ are obtained from $\varphi$ by (1) adding in all possible ways atoms $x=y, x \neq y, x \in \alpha$, and $x \notin \alpha$ until the obtained formula is explicit and then, (2) if a formula contains $x=y$, by removing atoms $P_{\alpha}(x, y, \vec{z})$ together with all occurrences of $\alpha$ in the sharing constraints (e.g., every atom $x \in \alpha$ or $\beta \subseteq \alpha$, where $\beta$ indexes a constraint $Q_{\beta}(u, v, \vec{w})$ and $u \neq v$ belongs to the formula, is replaced by false).

The equivalent formula $\psi_{1} \vee \ldots \vee \psi_{n}$ can be used to compute the normal form of $\varphi$ as follows. An atom $x=y$ or $x \neq y$ is implied by $\varphi$ iff this atom is included in all the 
satisfiable formulas $\psi_{i}$. Also, for any $P(x, y, \vec{z})$ in $\varphi$, there exists a model $(C, J)$ of $\varphi$ s.t. $S^{C}(x) \neq S^{C}(y)$ iff this atom is included in some satisfiable $\psi_{i}$.

In general, the number of satisfiable formulas in the disjunction $\psi_{1} \vee \ldots \vee \psi_{n}$ may be exponential w.r.t. the size of $\varphi$. However, all these formulas can be represented symbolically as the satisfying assignments of a boolean formula, denoted by $F(\varphi)$.

In order to simplify the presentation, we give below the construction of $F(\varphi)$ only for $M O L L$ formulas where variables are of the same type; [10] gives the general case. $F(\varphi)$ is defined over the set of boolean variables $B \operatorname{Vars}(F(\varphi))$ defined in Tab. 1 .

Table 1. Definition of the set $B \operatorname{Vars}(F(\varphi))$ of boolean variables used in $F(\varphi)$

$$
\begin{aligned}
{[x=y] } & \text { for every } x, y \in \operatorname{LVars}(\varphi) \\
{[x, y, f] } & \text { for every atom } x \mapsto \theta \text { of } \varphi \text { with }(f, y) \in \theta \\
{\left[P_{\alpha}(x, y, \vec{z})\right] } & \text { for every atom } P_{\alpha}(x, y, \vec{z}) \text { of } \varphi \\
{[x \in \alpha] } & \text { for every } x \in \operatorname{LVars}(\varphi) \text { and } \alpha \in \operatorname{Set} \operatorname{Vars}(\varphi)
\end{aligned}
$$

Given a satisfying assignment $\sigma: B \operatorname{Vars}(F(\varphi)) \rightarrow\{0,1\}$ for $F(\varphi)$ such that $\sigma([x, y, f])=1$, for any $[x, y, f] \in B \operatorname{Vars}(F(\varphi))$, we define the $M O L L$ formula $\psi_{\sigma}$ to be $\varphi$ to which the following transformations are applied:

- if $\sigma([x=y])$ is 0 , resp. 1 , then $\psi_{\sigma}$ includes the pure constraint $x \neq y$, resp. $x=y$,

- if $\sigma\left(\left[P_{\alpha}(x, y, \vec{z})\right]\right)=0$ then $P_{\alpha}(x, y, \vec{z})$ and $\alpha$ are removed from $\varphi$,

- if $\sigma([x \in \alpha])$ is 0 , resp. 1 , then $x \notin \alpha$, resp. $x \in \alpha$, is added to $\psi_{\sigma}$.

Let $\varphi=\Pi \wedge \Sigma \wedge \Lambda$ be a $M O L L$ formula. The formula $F(\varphi)$ is defined by:

$$
F(\varphi)=F(\Pi) \wedge F_{e q} \wedge F(\Sigma) \wedge F_{\text {det }} \wedge F(\Lambda) \wedge F_{\epsilon},
$$

where $F(\Pi), F(\Sigma)$, and $F(\Lambda)$ encode the semantics of the atomic formulas of $\varphi, F_{e q}$ encodes the reflexivity and the transitivity of the equality relation in $\Pi, F_{d e t}$ encodes the semantics of the field separating conjunction, and $F_{\in}$ encodes the properties of the membership relation $\in$. These sub-formulas are defined inductively on the syntax of MOLL formulas. Most of them are not difficult to follow. We provide here some intuition for the most interesting ones.

In $F(\Sigma)$, an atom $P_{\alpha}(x, y, \vec{z})$ is translated into $F\left(P_{\alpha}(x, y, \vec{z})\right)=\left[P_{\alpha}(x, y, \vec{z})\right] \oplus[x=$ $y$ ], where $\oplus$ is the exclusive or. This expresses the fact that the atom is kept in a reduced, explicit $M O L L$ formula only if its endpoints are not equal.

The separation of fields (defined for locations which are interpretations of location variables) induced by the use of the field separating conjunction is expressed in the formula $F_{d e t}$ in Fig. 8. Thus, $F_{\text {det }}$ states that for any location variable $x$ and any field $f \in$ Flds, at most one of the following conditions is true:

1. the reduced, explicit formula contains the equality $x=x^{\prime}$ and a points-to constraint $x^{\prime} \mapsto \theta$ such that $(f, y) \in \theta$, for some $y$,

2. the reduced, explicit formula contains the atoms $x \in \alpha$ and $P_{\alpha}\left(x^{\prime}, y, \vec{z}\right)$ (therefore it also includes $x^{\prime} \neq y$ ), for some $y$ and $\vec{z}$, such that $f \in \operatorname{Flds}_{0}\left(P_{\alpha}\right)$. 


$$
\begin{aligned}
F_{\text {det }}= & \bigwedge \text { for any }\left[x_{1}, y_{1}, f\right],\left[x_{2}, y_{2}, f\right] \in B \operatorname{Vars}(F(\varphi)) \text { different variables } \\
& {\left[x_{1}=x_{2}\right] \wedge\left[x_{1}, y_{1}, f\right] \Rightarrow \neg\left[x_{2}, y_{2}, f\right] } \\
& \bigwedge \text { for any }\left[x_{1}, y_{1}, f\right],\left[P_{\alpha}\left(x_{2}, y_{2}, \overrightarrow{z_{2}}\right)\right] \in B \operatorname{Vars}(F(\varphi)) \text { s.t. } f \in F l s_{0}(P) \text { and } x \in \operatorname{LVars}(\varphi) \\
& {\left[x_{1}=x\right] \wedge[x \in \alpha] \wedge\left[x_{1}, y_{1}, f\right] \Rightarrow \neg\left[P_{\alpha}\left(x_{2}, y_{2}, \overrightarrow{z_{2}}\right)\right] } \\
& \bigwedge \text { for any }\left[P_{\alpha}\left(x_{1}, y_{1}, \overrightarrow{z_{1}}\right)\right],\left[Q_{\beta}\left(x_{2}, y_{2}, \overrightarrow{z_{2}}\right)\right] \in B \operatorname{Vars}(F(\varphi)) \text { different variables } \\
& \text { s.t. } F l d s_{0}(P) \cap F l d s_{0}(Q) \neq \emptyset \text { and } x, x^{\prime} \in \operatorname{LVars}(\varphi) \\
& {[x \in \alpha] \wedge\left[x^{\prime} \in \beta\right] \wedge\left[x=x^{\prime}\right] \wedge\left[P_{\alpha}\left(x_{1}, y_{1}, \overrightarrow{z_{1}}\right)\right] \Rightarrow \neg\left[Q_{\beta}\left(x_{2}, y_{2}, \overrightarrow{z_{2}}\right)\right] }
\end{aligned}
$$

Fig. 8. Definition of $F_{d e t}$ for a $M O L L$ formula $\varphi=\Pi \wedge \Sigma \wedge \Lambda$

Fig. 9gives the main definitions of $F(\Lambda)$. For instance, $F\left(\alpha_{1} \subseteq \alpha_{2}\right)$ in eq. (14) expresses the fact that if there exists some variable $x$ such that $x \in \alpha_{1}$ is true then $x \in \alpha_{2}$ also holds. In eq. (15), $F_{\in}$ encodes the closure of $\in$ under the equality, the fact that if a boolean variable $\left[x_{1} \in \alpha\right]$ is true then the list segment bound to $\alpha$ in $\varphi$, if any, is not empty, and if $\alpha$ is bound to a non-empty list segment $P_{\alpha}(x, y, \vec{z})$ in $\varphi$, then $\alpha$ contains the first element of the segment, i.e., $x$.

$$
\begin{aligned}
F\left(x \in \alpha_{1}\right) & =\left[x \in \alpha_{1}\right] \\
F\left(x \in \bigcup_{1 \leq i \leq n}\left\{u_{i}\right\}\right)= & \bigvee_{1 \leq i \leq n}\left[x=u_{i}\right] \\
F\left(\alpha_{1} \subseteq \alpha_{2}\right)= & \bigwedge_{x \in \operatorname{Lars}(\varphi)}\left[x \in \alpha_{1}\right] \Rightarrow\left[x \in \alpha_{2}\right] \\
F_{\in}= & \bigwedge_{u, v, \alpha \operatorname{in} \varphi}([u=v] \wedge[u \in \alpha]) \Rightarrow[v \in \alpha] \\
& \wedge \bigwedge_{x_{1}, P_{\alpha}(x, y, \vec{z}) \text { in } \varphi}\left(\left[x_{1} \in \alpha\right] \Rightarrow\left[P_{\alpha}(x, y, \vec{z})\right]\right) \wedge\left(\left[P_{\alpha}(x, y, \vec{z})\right] \Rightarrow[x \in \alpha]\right)
\end{aligned}
$$

Fig. 9. Main definitions of $F(\Lambda)$ and $F_{\in}$ for a $M O L L$ formula $\varphi=\Pi \wedge \Sigma \wedge \Lambda$

Proposition 1. The size of $F(\varphi)$ is polynomial in the size of $\varphi$.

Proposition 2. Let $\varphi$ be a NOLL formula. For any satisfying assignment $\sigma$ of $F(\varphi), \psi_{\sigma}$ is an explicit, reduced, and satisfiable formula. Also, $\varphi$ is equivalent to the disjunction of $\psi_{\sigma}$, for all satisfying assignments $\sigma$ of $F(\varphi)$.

Theorem 4. The problem of computing the normal form of a formula $\varphi$ is in co-NP.

Proof. To compute the maximum set of (in)equalities that should be included in the normal form of $\varphi$, we iterate over every pair of location variables $x, y$ in $\varphi$ and check if $F(\varphi) \Rightarrow[x=y]$ or $F(\varphi) \Rightarrow \neg[x=y]$ is valid. In the first (resp., second) case, $x=y$ (resp., $x \neq y$ ) is included in the normal form. When some equality $x=y$ is added to the normal form, the atoms $P_{\alpha}(x, y, \vec{z})$ in $\varphi$ are removed, and all occurrences of $\alpha$ are interpreted as the empty set. Since we need to perform a polynomial number of Boolean formula validity tests, the overall complexity of this procedure is co-NP time. 


\section{An Effective Procedure for Checking Entailment}

The procedure for checking the validity of the entailments $\varphi \Rightarrow \psi$ between two NOLL formulas is detailed in Fig. 10 It has three main steps:

procedure CheckEntl $(\varphi \Rightarrow \psi)$

1: $\varphi^{\prime}:=$ the normal form of $\varphi$

2: $\psi^{\prime}:=$ the normal form of $\psi$

3: $G_{1}:=$ the complete NOLL graph of $\varphi^{\prime}$

4: $G_{2}:=$ the NOLL graph of $\psi^{\prime}$

5: $h:=$ the function $h: V\left(G_{2}\right) \rightarrow V\left(G_{1}\right)$ s.t. $\operatorname{vars}_{G_{2}}(n) \subseteq \operatorname{vars}_{G_{1}}(h(n)), \forall n \in V\left(G_{2}\right)$

6: return ( $h$ is total) and

( $h$ is a homomorphism)

Fig. 10. (a) compute (lines 1-2) the normal form of $\varphi$ and $\psi$, denoted by $\varphi^{\prime}$ and $\psi^{\prime}$, respectively, (b) compute (line 3) additional spatial constraints, which are implied by $\varphi$, and (c) check (lines 3-6) if the graph representation of $\psi^{\prime}$ is homomorphic to the graph representation of both $\varphi^{\prime}$ and the additional constraints computed in the previous step.

In the following, we first de-

scribe the step (b) above, then we define graph representations for $N O L L$ formulas, called (complete) NOLL graphs, and finally, we define the notion of homomorphism between NOLL graphs. Moreover, we assume that $\varphi$ and $\psi$ are satisfiable. Otherwise, Proposition 2 implies that a formula $\varphi$ is satisfiable iff $F(\varphi)$ is satisfiable, which allows to decide in co-NP time entailments of the form $\varphi \Rightarrow \psi$ when $\varphi$ or $\psi$ is unsatisfiable.

\subsection{Inferring Additional Spatial Constraints}

In order to give an intuition about the additional spatial constraints deduced from $\varphi$, recall the entailment $\varphi_{1} \Rightarrow \varphi_{2}$, where $\varphi_{1}$ and $\varphi_{2}$ are defined in eq. (6-7) at page 134 The entailment holds because the list segments linking $x$ to $n$ and $n$ to $y$, and described by $\operatorname{List}_{\delta}(x, n) * \operatorname{List}_{\gamma}(n, y)$, exist in every model of $\varphi_{1}$. To obtain a complete decision procedure for entailment, such constraints must be made explicit before checking the existence of a homomorphism between the two formulas viewed as graphs.

Observe that $\varphi_{1}$ does not imply $\varphi_{1} *_{w}\left(\operatorname{List}_{\delta}(x, n) * \operatorname{List}_{\gamma}(n, y)\right)$ but, $\varphi_{1} \wedge\left(\operatorname{List}_{\delta}(x, n) *\right.$ $\left.\operatorname{List}_{\gamma}(n, y)\right)$. Thus, these implicit constraints will be added only to the graph representation of NOLL formulas and not to the formula itself, as explained in Sec.6.2

For simplicity, we give the definition only for MOLL formulas $\varphi$. Let $\xi$ be a set of atoms in $\varphi$ of the form $Q_{\beta}(u, v, \vec{w})$. For any such $\xi, \mathcal{P}(\xi)$ denotes the set of recursive predicates in $\xi$, SetVars $(\xi)$ denotes the set of variables $\beta \in$ SetVars bounded to atoms in $\xi$, and $t_{\xi}$ is the term defined as the union of all variables in $\operatorname{Set} \operatorname{Vars}(\xi)$.

An atom $P_{\alpha}(x, y, \vec{z})$ is called implicit in $\xi$ iff one of the following holds:

- $\xi$ consists of one atom $P_{\beta}(u, v, \vec{z})$, the source of $P_{\alpha}$ is the same as the source of $P_{\beta}$, i.e., $\varphi \Rightarrow x=u$, and the destination of $P_{\alpha}$ is included in the list segment defined by $P_{\beta}$, i.e., $\varphi \Rightarrow y \in \beta$;

- (1) $\varphi \Rightarrow x \in t_{\xi}$, (2) $t_{\xi}$ is a minimal term $t$ such that $\varphi \Rightarrow x \in t$, i.e., for every other term $t^{\prime}$, which is the union of the variables from a strict subset of $\operatorname{SetVars}(\xi), \varphi \nRightarrow x \in t^{\prime}$, (3) $F l d s_{0}(P)=\bigcap_{Q \in \mathcal{P}(\xi)} F l d s_{0}(Q)$, and (4) $\varphi \Rightarrow \bigwedge_{Q_{\beta}(u, v, \vec{z}) \in \xi} y=v$.

Similarly, an atom $x \mapsto\{(f, y)\}$ is called implicit in $\xi$ iff the conditions (1) and (2) above hold, ( $\left.3^{\prime}\right)$ an atom $u \mapsto \theta_{i}$ with $\left(f, d_{i}\right) \in \theta_{i}$ is included in the definition of $Q$, for all $Q \in \mathcal{P}(\xi)$, and $\left(4^{\prime}\right) \varphi \Rightarrow \bigwedge_{1 \leq i \leq n} y=d_{i}$. 
For example, for $\xi=\left\{\operatorname{List}_{\alpha}(x, y)\right\}$ a set of atoms in $\varphi_{1}$ from eq. (6), the atom List $_{\delta}(x, n)$ is implicit in $\xi$ because $\beta \subseteq \alpha$ in $\varphi_{1}$ implies that $n \in \alpha$ and the equality $x=x$ is trivially implied by $\varphi_{1}$. Also, the atom $\operatorname{List}_{\gamma}(n, y)$ is implicit in $\xi$ because the conditions (1-4) above hold.

By definition, the Boolean abstraction $F(\varphi)$ defined in Sec. 5 can be used to check that $\varphi$ implies the equalities and the sharing constraints in the above conditions. The conditions (3) and $\left(3^{\prime}\right)$ can be checked syntactically. Thus, the computation of the implicit spatial constraints for a formula is co-NP complete.

\subsection{NOLL Graphs}

We define NOLL graphs, a graph representation for NOLL formulas. Roughly, the nodes of these graphs represent sets of equal location variables and the edges represent spatial or difference constraints. The object separated spatial constraints are represented by a binary relation $\Omega_{*}$ over edges while the sharing constraints are kept unchanged.

Definition 3 (NOLL graph). Given a NOLL formula $\varphi=\Pi \wedge \Sigma \wedge \Lambda$ over a set of predicates $\mathcal{P}$, the NOLL graph of $\varphi$, denoted $G(\varphi)$, is a tuple $\left(V, E_{P}, E_{R}, E_{D}, \ell, \Omega_{*}, \Lambda\right)$ or the error graph $\perp$, where:

- each node in $V$ denotes an equivalence class over elements of LVars w.r.t. the equality relation defined in $\Pi$; the equivalence class of $x$ is denoted by $[x]$. If $\Pi$ contains both $x \neq y$ and $x=y$ then $G$ is the error graph $\perp$;

- $E_{P} \subseteq V \times$ Flds $\times V$ represents the points-to constraints: $([x], f,[y]) \in E_{P}$ iff $x \mapsto \theta$ with $(f, y) \in \theta$ is an atomic formula in $\Sigma$;

- $E_{R} \subseteq V \times \mathcal{P} \times V^{+} \times V$ represents list segment constraints: $\left([x], P_{\alpha},[\vec{z}],[y]\right) \in E_{R}$ iff $P_{\alpha}(x, y, \vec{z})$ is an atomic formula in $\Sigma$;

- $E_{D} \subseteq V \times V$ represents inequalities: $([x],[y]) \in E_{D}$ iff $x \neq y$ is an atom in $\Pi$;

- $\ell:$ LVars $\rightarrow V$, called variable labeling, it is defined by $\ell(x)=[x]$, for any $x \in$ LVars;

- $\Omega_{*}$ contains all pairs of edges in $E_{P} \cup E_{R}$ denoting object separated atoms in $\Sigma$.

In the following, $V(G)$, denotes the set of nodes in the NOLL graph $G$. We use a similar notation for all the other components of $G$. Also, for any $n \in V(G), \operatorname{vars}_{G}(n)$ denotes the set of all the variables labeling the node $n$ in $G$. The graph $G\left(\varphi_{2}\right)$ in Fig. 3 represents the NOLL graph of $\varphi_{2}$, where $V=\{x, y, n, m\}, E_{P}=E_{D}=\emptyset, E_{R}$ contains the three edges corresponding to the three list segments, $\Omega_{*}$ contains only one pair $\left\langle\left([x]\right.\right.$, List $\left._{\alpha},[n]\right),\left([n]\right.$, List $\left.\left._{\beta^{\prime}},[y]\right)\right\rangle$, and $\Lambda$ is $\beta^{\prime} \subseteq \delta \cup \gamma$.

A graph representation for $\varphi$ which includes an edge for each implicit spatial constraint of $\varphi$ is called a complete NOLL graph. This representation has an additional attribute $\Delta$, which identifies the set of atoms where a spatial constraint is implicit in.

Definition 4 (complete NOLL graph). Given a NOLL formula $\varphi=\Pi \wedge \Sigma \wedge \Lambda$, the complete NOLL graph of $\varphi$, denoted by $\bar{G}(\varphi)$ is a tuple $(G, \Delta)$ where:

- $G$ is a NOLL graph where all components except $E_{R}, E_{P}, \Omega_{*}$, and $\Lambda$ are equal to the components of $G(\varphi)$;

- $E_{R}(G)$ (resp. $E_{P}(G)$ ) includes $E_{R}(G(\varphi))$ (resp. $E_{P}(G(\varphi))$ ) and, for any atom $P_{\alpha}(x, y, \vec{z})$ (resp. $x \mapsto\{(f, y)\}$ ) which is implicit in some set of atoms $\xi, e=$ $\left([x], P_{\alpha},[\vec{z}],[y]\right) \in E_{R}(G)$ (resp. $\left.e=([x], f,[y]) \in E_{P}(G)\right)$; 
- $\Omega_{*}(G)$ consists of $\Omega_{*}(G(\varphi))$ plus all pairs $\left(e, e^{\prime}\right)$ s.t. e represents an implicit constraint in $\xi$ and $\left(e^{\prime}, e^{\prime \prime}\right) \in \Omega_{*}(G)$ for some $e^{\prime \prime}$ representing an atom in $\xi$;

- $\Delta \subseteq\left(E_{P} \cup E_{R}\right) \times 2^{E_{R}}$ represents the relation between edges and the sets of list segments where they are implicit in, i.e., for every $P_{\alpha}(x, y, \vec{z})$ (resp. $\left.x \mapsto\{(f, y)\}\right)$ implicit in $\xi,\left(\left([x], P_{\alpha},[\vec{z}],[y]\right), E_{\xi}\right) \in \Delta$ (resp. $\left.\left(([x], f,[y]), E_{\xi}\right) \in \Delta\right)$, where $E_{\xi}$ is the set of edges representing the atoms in $\xi$;

- if $P_{\alpha_{1}}(x, y, \vec{z})$ and $P_{\alpha_{2}}(y, t, \vec{z})$ are implicit in $\xi=\left\{P_{\alpha}(x, t, \vec{z})\right\}$ then, $\alpha=\alpha_{1} \cup \alpha_{2}$ is added to $\Lambda$.

The graph in the middle of Fig. 3 represents the complete NOLL graph of $\varphi_{1}, \bar{G}\left(\varphi_{1}\right)$, where $V=\{x, y, n, m\}, E_{P}=E_{D}=\Omega_{*}=\emptyset$, and $E_{P}$ contains the four edges: two edges represent the spatial constraints in $\varphi_{1}$, and the edges $\left([x]\right.$, List $\left._{\alpha_{1}},[n]\right)$ and $\left([n]\right.$, List $\left._{\alpha_{2}},[m]\right)$ represent implicit constraints in $\xi=\left\{\operatorname{List}_{\alpha}(x, y)\right\} . \Lambda$ is $\beta \subseteq \alpha \wedge \alpha=\alpha_{1} \cup \alpha_{2}$ and $\Delta$ is the relation $\left\{\left(\left([x]\right.\right.\right.$, List $\left.\left._{\alpha_{1}},[n]\right), \xi\right),\left(\left([n]\right.\right.$, List $\left.\left.\left._{\alpha_{2}},[m]\right), \xi\right)\right\}$.

\subsection{NOLL Graph Homomorphism}

Given a NOLL graph $G_{1}$ and a complete $N O L L$ graph $G_{2}$, a homomorphism from $G_{1}$ to $G_{2}$ is a mapping $h: V\left(G_{1}\right) \mapsto V\left(G_{2}\right)$, which:

1. preserves the labeling with location variables, i.e., $\operatorname{vars}_{G_{1}}(n) \subseteq \operatorname{vars}_{G_{2}}(h(n))$, for any $n \in V\left(G_{1}\right)$,

2. maps each difference, resp., points-to, edge of $G_{1}$ to a difference, resp., points-to, edge of $G_{2}$, (e.g., for any $\left.\left(n, f, n^{\prime}\right) \in E_{P}\left(G_{1}\right),\left(h(n), f, h\left(n^{\prime}\right)\right) \in E_{P}\left(G_{2}\right)\right)$,

3. maps each edge representing a list segment in $G_{1}$ to a path in $G_{2}$ formed of edges in $E_{P}\left(G_{2}\right) \cup E_{R}\left(G_{2}\right)$, and

4. satisfies the constraints required by the semantics of the separating conjunctions, the special status of the implicit spatial constraints, and the sharing constraints.

To explain the mapping of edges in $E_{R}\left(G_{1}\right)$ to paths of $G_{2}$, let us consider the case of an edge $\left(n, P_{\alpha}, m, p\right) \in E_{R}\left(G_{1}\right)$, where $n, m, p \in V\left(G_{1}\right)$ and $P$ is a MOLL predicate, i.e., $P($ in, out,$b) \triangleq($ in $=$ out $) \vee\left(\exists u . \Sigma_{0}(\right.$ in,$u \cup b) * P(u$, out,$\left.b)\right)$. The definition of $h$ requires that there exists a sequence of nodes $\pi=\pi_{1} \ldots \pi_{k}, k \geq 1$, in $G_{2}$ s.t. $\pi_{1}=h(n), \pi_{k}=h(p)$, and for every two consecutive nodes $\pi_{i}$ and $\pi_{i+1}$, either

- $E_{P}\left(G_{2}\right)$ contains some set of edges between $\pi_{i}, \pi_{i+1}$, and $h(m)$, which prove that $\Sigma_{0}\left(x_{i}, x_{i+1} \cup x_{h(m)}\right)$ holds, where $x_{i}, x_{i+1}$, and $x_{h(m)}$ are some variables labeling $\pi_{i}$, $\pi_{i+1}$, and $h(m)$, respectively, or

- there exists an edge $\left(\pi_{i}, P_{\beta}^{\prime}, \vec{q}, \pi_{i+1}\right)$ in $E_{R}\left(G_{2}\right)$, representing a stronger predicate than $P_{\alpha}$, i.e., $h(m) \in \vec{q}$ and $P_{\beta}^{\prime}\left(x_{i}, x_{i+1}, \vec{z}\right) \Rightarrow P_{\alpha}\left(x_{i}, x_{i+1}, x_{h(m)}\right)$, where $x_{i}, x_{i+1}$, and $x_{h(m)}$ are as above, and $\vec{z}$ is a set of variables labeling $\vec{q}$ s.t. $x_{h(m)} \in \vec{z}$ (this is possible because $h(m) \in \vec{q}$ ). The entailment between recursive predicates can be checked syntactically in polynomial time.

In the following, we explain the constraints required by the $4^{\text {th }}$ item in the definition of the homomorphism. For any edge $e$ in $E_{P}\left(G_{1}\right) \cup E_{R}\left(G_{1}\right)$, we define a set used $(e) \subseteq$ $E_{P}\left(G_{2}\right) \cup 2^{\left(E_{R}\left(G_{2}\right) \times F l d s\right)}$, which represents all the edges/fields used in the path from $G_{2}$ to which $e$ is mapped by $h$. If $e \in E_{P}\left(G_{1}\right)$ then $\operatorname{used}(e)=\left\{e^{\prime}\right\}$, where $e^{\prime}$ is the edge of 
$G_{2}$ to which $e$ is mapped by $h$. If $e \in E_{R}\left(G_{1}\right)$ represents a list segment $P_{\alpha}$ then, used $(e)$ consists of (1) the set of points-to edges in the path associated to $e$ and (2) the set of pairs $\left(e^{\prime}, f\right)$, where $e^{\prime}$ represents a list segment $Q_{\beta}$ from the same path, if such an edge exists, and $f \in \operatorname{Flds}_{0}(P) \cap F l d s_{0}(Q)$. When the path associated to $e \in E_{R}\left(G_{1}\right)$ labeled by $P_{\alpha}$ (resp. $e \in E_{P}\left(G_{1}\right)$ labeled by $f$ ) contains an edge $e^{\prime}$ representing a constraint implicit in some $\xi$, i.e., $\left(e^{\prime}, E_{\xi}\right) \in \Delta\left(G_{2}\right)$, then used $(e)$ includes all pairs $\left(e^{\prime \prime}, f\right)$ with $e^{\prime \prime} \in E_{\xi}$ labeled by $Q_{\beta} \in \xi$, and $f \in$ Fld $_{0}(P) \cap$ Fld $_{0}(Q)$ (resp. $f \in$ Fld $_{0}(Q)$ ).

Then, to express the semantics of $*_{w}$, we require that used $\left(e_{1}\right) \cap \operatorname{used}\left(e_{2}\right)=\emptyset$, for any two edges $e_{1}$ and $e_{2}$ in $E_{P}\left(G_{1}\right) \cup E_{R}\left(G_{1}\right)$. Concerning $*$, it is required that for any two edges $e_{1}$ and $e_{2}$ in $E_{P}\left(G_{1}\right) \cup E_{R}\left(G_{1}\right)$ s.t. $\left(e_{1}, e_{2}\right) \in \Omega_{*}\left(G_{1}\right)$, we have that $\left(e_{1}^{\prime}, e_{2}^{\prime}\right) \in$ $\Omega_{*}\left(G_{2}\right)$, for any $e_{1}^{\prime}$ an edge appearing in $\operatorname{used}\left(e_{1}\right)$ and $e_{2}^{\prime}$ an edge appearing in used $\left(e_{2}\right)$.

Finally, for the sharing constraints, the mapping by $h$ of edges in $E_{R}\left(G_{1}\right)$ to paths in $G_{2}$ defines a substitution $\Gamma$ for set of locations variables in $\Lambda\left(G_{1}\right)$ to terms over set of locations variables in $\Lambda\left(G_{2}\right)$. For example, the homomorphism in Fig. 3 defines the substitution $\Gamma(\delta)=\alpha_{1}, \Gamma(\gamma)=\alpha_{2}$, and $\Gamma\left(\beta^{\prime}\right)=\beta$. Then, it is required that $\Lambda\left(G_{2}\right) \Rightarrow$ $\Lambda\left(G_{1}\right)[\Gamma]$. Such a formula belongs for instance, to the fragment of BAPA [14], and thus its validity can be decided in NP-time. For the example in Fig. 3, we obtain the trivial entailment $\beta \subseteq \alpha \wedge \alpha=\alpha_{1} \cup \alpha_{2} \Rightarrow \beta \subseteq \alpha_{1} \cup \alpha_{2}$.

\subsection{Checking Entailments of NOLL Formulas}

The following theorem states the correctness and the complexity of the procedure CheckEntl given in Fig. 10, the proof is given in [10].

Theorem 5. Given two NOLL formulas $\varphi$ and $\psi, \varphi \Rightarrow \psi$ holds iff CheckEnt $(\varphi \Rightarrow \psi)$ returns true. Moreover, the complexity of CheckEntl is co-NP time.

\section{Experimental Results}

We have implemented the procedure for entailment checking in a solver which takes as input the specification of predicates in $\mathcal{P}$ and two formulas $\varphi, \psi \in N O L L$ defined over $\mathcal{P}$ and returns as result either the homomorphism found when $\varphi \Rightarrow \psi$ or a diagnosis explaining why the entailment is not valid. The diagnosis is given as a list of variables or atomic spatial constraints in $\varphi$ and $\psi$ for which the conditions for the homomorphism are not satisfied. The solver is implemented in C. It uses MiniSat [9] to compute normal forms and an ad-hoc solver for the sharing constraints.

We have used this solver to check verification conditions generated for procedures working on singly linked lists, doubly linked lists, and overlaid hash tables and lists in the Nagios network monitoring example. We have considered mainly the procedures for inserting or moving elements in these data structures. The post-condition computation follows the standard approach: introducing primed variables to denote old values and unfolding recursive predicates for statements that involve fields. To generate simpler verification conditions, we use the frame rules for the separating conjunction operators. In this way, the graph representations for the NOLL formulas have less than ten vertices and twenty edges (including the inferred edges), and less than five set of locations variables. Each verification condition is decided in less than 0.1 seconds. 


\section{References}

1. Berdine, J., Calcagno, C., Cook, B., Distefano, D., O'Hearn, P.W., Wies, T., Yang, H.: Shape Analysis for Composite Data Structures. In: Damm, W., Hermanns, H. (eds.) CAV 2007. LNCS, vol. 4590, pp. 178-192. Springer, Heidelberg (2007)

2. Berdine, J., Calcagno, C., O’Hearn, P.W.: A Decidable Fragment of Separation Logic. In: Lodaya, K., Mahajan, M. (eds.) FSTTCS 2004. LNCS, vol. 3328, pp. 97-109. Springer, Heidelberg (2004)

3. Berdine, J., Calcagno, C., O'Hearn, P.W.: Smallfoot: Modular Automatic Assertion Checking with Separation Logic. In: de Boer, F.S., Bonsangue, M.M., Graf, S., de Roever, W.-P. (eds.) FMCO 2005. LNCS, vol. 4111, pp. 115-137. Springer, Heidelberg (2006)

4. Bobot, F., Filliâtre, J.-C.: Separation Predicates: A Taste of Separation Logic in FirstOrder Logic. In: Aoki, T., Taguchi, K. (eds.) ICFEM 2012. LNCS, vol. 7635, pp. 167-181. Springer, Heidelberg (2012)

5. Calcagno, C., Yang, H., O'Hearn, P.W.: Computability and Complexity Results for a Spatial Assertion Language for Data Structures. In: Hariharan, R., Mukund, M., Vinay, V. (eds.) FSTTCS 2001. LNCS, vol. 2245, pp. 108-119. Springer, Heidelberg (2001)

6. Chang, B.-Y.E., Rival, X.: Relational inductive shape analysis. In: POPL, pp. 247-260. ACM (2008)

7. Chlipala, A.: Mostly-automated verification of low-level programs in computational separation logic. In: PLDI, pp. 234-245. ACM (2011)

8. Cook, B., Haase, C., Ouaknine, J., Parkinson, M., Worrell, J.: Tractable Reasoning in a Fragment of Separation Logic. In: Katoen, J.-P., König, B. (eds.) CONCUR 2011. LNCS, vol. 6901, pp. 235-249. Springer, Heidelberg (2011)

9. Eén, N., Sörensson, N.: An Extensible SAT-solver. In: Giunchiglia, E., Tacchella, A. (eds.) SAT 2003. LNCS, vol. 2919, pp. 502-518. Springer, Heidelberg (2004)

10. Enea, C., Saveluc, V., Sighireanu, M.: Composite invariant checking for nested, overlaid linked lists (2012), Extended version available as HAL-00768389 report

11. Hawkins, P., Aiken, A., Fisher, K., Rinard, M.C., Sagiv, M.: Data representation synthesis. In: PLDI, pp. 38-49. ACM (2011)

12. Ishtiaq, S., O'Hear, P.W.: BI as an assertion language for mutable data structures. In: POPL, pp. 14-26. ACM (2001)

13. Jacobs, B., Smans, J., Piessens, F.: A Quick Tour of the VeriFast Program Verifier. In: Ueda, K. (ed.) APLAS 2010. LNCS, vol. 6461, pp. 304-311. Springer, Heidelberg (2010)

14. Kuncak, V., Nguyen, H.H., Rinard, M.: An Algorithm for Deciding BAPA: Boolean Algebra with Presburger Arithmetic. In: Nieuwenhuis, R. (ed.) CADE 2005. LNCS (LNAI), vol. 3632, pp. 260-277. Springer, Heidelberg (2005)

15. Madhusudan, P., Qiu, X., Stefanescu, A.: Recursive proofs for inductive tree data-structures. In: POPL, pp. 123-136. ACM (2012)

16. Navarro Pérez, J.A., Rybalchenko, A.: Separation logic + superposition calculus $=$ heap theorem prover. In: PLDI, pp. 556-566. ACM (2011)

17. Reynolds, J.C.: Separation logic: A logic for shared mutable data structures. In: LICS, pp. 55-74. IEEE Computer Society (2002)

18. Suter, P., Dotta, M., Kuncak, V.: Decision procedures for algebraic data types with abstractions. In: POPL, pp. 199-210. ACM (2010)

19. Yang, H., Lee, O., Berdine, J., Calcagno, C., Cook, B., Distefano, D., O’Hearn, P.W.: Scalable Shape Analysis for Systems Code. In: Gupta, A., Malik, S. (eds.) CAV 2008. LNCS, vol. 5123, pp. 385-398. Springer, Heidelberg (2008) 